\title{
Psychological stress and the passage of a standard meal through the stomach and small intestine in man
}

\author{
P A CANN, N W READ, J CAMMACK, H CHILDS, S HOLDEN, R KASHMAN, \\ J LONGMORE, S NIX, N SIMMS, K SWALLOW, AND J WELLER
}

From the Department of Physiology, University of Sheffield, and Gastrointestinal Unit, Royal Hallamshire Hospital, Sheffield

SUMMARY Gastric emptying half-time and mouth to caecum transit time of a solid meal were measured in eight normal volunteers, once during a period of psychological stress and again during a period of relative calm. No consistent or significant effect on gastric emptying was observed, but mouth to caecum transit times were faster in all subjects and this difference was highly significant $(\mathrm{p}<0 \cdot 01)$.

We have previously reported that the transit time of a meal through the small intestine in patients with irritable bowel syndrome varied according to the predominant disorder of bowel habit. ${ }^{1}$ Patients who presented with constipation had significantly delayed small bowel transit compared with normal volunteers, while in patients who presented with diarrhoea small bowel transit was accelerated significantly. Moreover, we found a clear temporal association between the entry of food into the colon and symptoms of abdominal pain and distension. These data suggest that disordered small bowel transit may play. an important role in the pathogenesis of irritable bowel syndrome.

The mechanism responsible for these findings is not clear, but may be because of, at least in part, the influence of psychological stress. Patients with irritable bowel syndrome exhibit anxiety levels and life stress scores which are significantly higher than those found in normal volunteers or other disease states, such as ulcerative colitis. ${ }^{2-7}$ When patients are subdivided, however, it is only those complaining of diarrhoea who exhibit significantly higher anxiety levels,${ }^{2}$ while those with constipation and pain exhibit anxiety levels which are not significantly higher than normal.

Previous studies have reported effects of stress or emotion on salivation, ${ }^{8}$ oesophageal motility, ${ }^{9}$ gastric motility and secretion, ${ }^{10-14}$ small bowel motility ${ }^{15-18}$ and colonic motility. ${ }^{19-20}$ Nevertheless, not all of these studies have been compared with

Received for publication 8 June 1982 appropriate control experiments or have used stress stimuli analogous to the psychological stress patients often experience. Moreover, few have studied the influence of a prolonged period of maintained stress. In the present study we have investigated the effect of a controlled and prolonged stressful stimulus on measurements of gastric emptying and small bowel transit time.

\section{Methods}

\section{SUBJECTS}

Eight healthy students (seven women and one man) aged 19-21 years participated in the study. None of the subjects admitted to suffering from undue stress in their daily life and all had recently completed their terminal examinations with success. No medication was taken for at least 72 hours before each study, although three of the women were using oral contraceptives.

Each subject gave informed consent for the studies. The protocol was approved by the Ethical Subcommittee of the Sheffield Area Health Authority (Teaching) (Southern District). The women included in their consent a statement to the effect that to their knowledge they were not pregnant, neither were they likely to become pregnant during the period of the study.

\section{STUDY DESIGN}

Two studies were carried out on each subject. The first study always included the stress stimulus while the second was the control. This order was adopted so that the effect of the stress was reinforced with 
any stress induced by the novelty of the procedure. We have previously found, however, that repeat values for small bowel transit time are reproducible and show no consistent trend between first and second studies in normal volunteers ${ }^{21}$ or in patients with irritable bowel syndrome (unpublished observations). Both studies started at the same time of day ${ }^{22}$ and lasted for an equivalent period. There was an interval of one day between the two studies.

The subjects starved for at least nine hours before each study and were then given the standard test meal, consisting of Frankfurter sausages, mashed potato, baked beans, pineapple custard, and a drink of $50 \mathrm{ml}$ of water. ${ }^{21}$ Immediately after ingestion of the meal the subject laid supine on a comfortable bed and remained so for eight hours. During the first four hours they listened to either the stress or control tape recording through stereo headphones. Measurements of gastric emptying and small bowel transit time were recorded at 10 minute intervals and measurements of pulse rate, respiratory rate, and blood pressure were recorded at 20 finute intervals throughout the eight-hour period. Subjects worked in pairs, one undergoing a study, while the other measured and recorded the physiological data. Thus, measurements on each subject were made by the same observer during control and stress experiments. The subjective levels of anxiety, cooperation and alertness during each experiment were indicated by each subject on visual analogue scales ${ }^{23}$ on four occasions during the study; before eating the meal and at one-and-a-half, three and four hours afterwards.

\section{INDUCTION OF STRESS}

Stress was induced by means of a dichotomous listening test. ${ }^{1624} \mathrm{~A}$ stereo tape recording was constructed in such a way as to produce one spoken essay in one ear and a different essay in the other ear of a pair of headphones simultaneously. All recordings were made by the same speaker and material was taken from the following works: J Bronowski. The ascent of man. London: $\mathrm{BBC}$ Publications, 1973. A J P Taylor. How wars begin. London: Hamish Hamilton, 1977. D Morris. Manwatching. London: Jonathan Cape, 1977.

Bells and buzzers were incorporated into the stress recording, randomly both in time, and from ear to ear. After each buzzer, the subject was required to write down the sixth word after it in the opposite ear. After each bell, they were told to answer the next question on a card in front of them, pertaining to information following the bell in the same ear.

The tape used for the control period was a mono recording of a light novel (L Lee. As I walked out one midsummer morning. Harmondsworth, Herts: Penguin Books, 1971) and was uninterrupted.

TRANSIT OF THE SOLID MEAI.

Gastric emptying and mouth to caecum transit time were measured using the technique described by Read et al. ${ }^{21}$ Fifty $\mu \mathrm{Cie}$ of ${ }^{99 \mathrm{~m}}$ technetium sulphur colloid was added to the test meal and gastric emptying was measured by monitoring the radioactive counts over the surface of the stomach using a crystal scintillation detector linked to a counter ratemeter. Mouth to caecum transit time was measured by monitoring the level of hydrogen gas in end expiratory breath samples, using a metallised membrane electrode. ${ }^{25-27}$ Small bowel transit time was taken as the time at which the first sustained rise in breath hydrogen was seen.

\section{STATISTICAL METHODS}

The degree of significance between paired measurements of transit, pulse and respiratory rates, and blood pressure were calculated using Student's $t$ test. Subjective assessments of anxiety, cooperation, and alertness were compared using Wilcoxon's signed rank sum tests.

\section{Results}

\section{ASSESSMENT OF STRESS}

Objective

It can be seen from Figure 1 that pulse rates, respiratory rates, and blood pressures were all higher during the stress stimulus compared with the control period and that the differences between most of the paired measurements of pulse, respiration, and systolic blood pressure were statistically significant, particularly during the first three hours of stress. After the tape recording finished at four hours there was a tendency for the paired measurements to become congruous. No correlation could be found between individual changes in these measurements and the changes in small bowel transit time or gastric emptying.

\section{Subjective}

All subjects reported an increased level of anxiety during stress compared with the control study and these differences were significant at the beginning of the test and at 90 minutes. There were no significant differences, however, in reported levels of cooperation and alertness between the two studies (Fig. 2).

\section{GASTRIC EMPTYING HALF-TIME}

The stomach emptied in an exponential fashion in all subjects during both control and stress studies. 


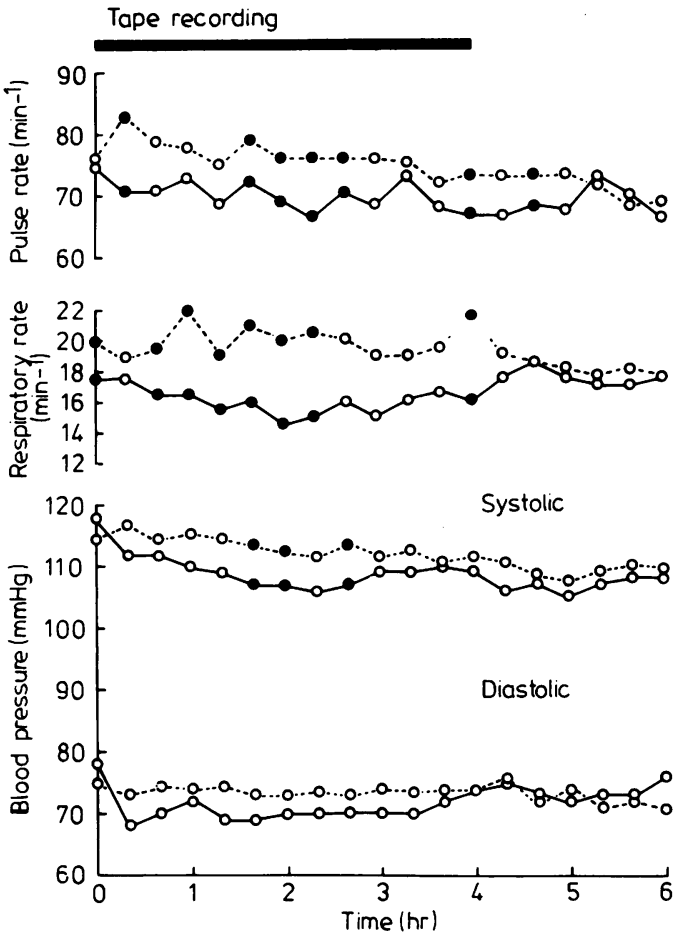

Fig. 1 Mean pulse rate, respiratory rate, and blood pressure for whole group during control (O_- $\left.)_{1}\right)$ and stress $(0 . . .-0)$ studies. Closed circles $(\bullet)$ significant difference $(p<0.05)$ between paired means. The duration of stress and control tape recordings is indicated by horizontal bar.

Gastric emptying was faster during stress in two subjects. slower in three subjects and unchanged in the remainder. No significant differences in halftimes for gastric emptying could be shown between the two studies (Table).

\section{MOUTH TO CAECUM TRANSIT TIME}

Mouth to caecum transit time was between three and $44 \%$ (mean $26 \%$ ) shorter during the stress study in all eight subjects $(\mathrm{p}<0.01)$ (Table).

It can be seen from Figure 3 that although the

Table Transit measurements for control and stress studies

\begin{tabular}{llll}
\hline & Control & Stress \\
\hline $\begin{array}{l}\text { Gastric emptying half-time } \\
\text { (minutes) }\end{array}$ & $112( \pm 3)$ & $110( \pm 6)$ & ns \\
$\begin{array}{l}\text { Mouth to caecum transit time } \\
\text { (minutes) }\end{array}$ & $381( \pm 49)$ & $276( \pm 33)$ & $\mathrm{p}<0.01$ \\
\hline
\end{tabular}

Results expressed as mean from group \pm SEM.

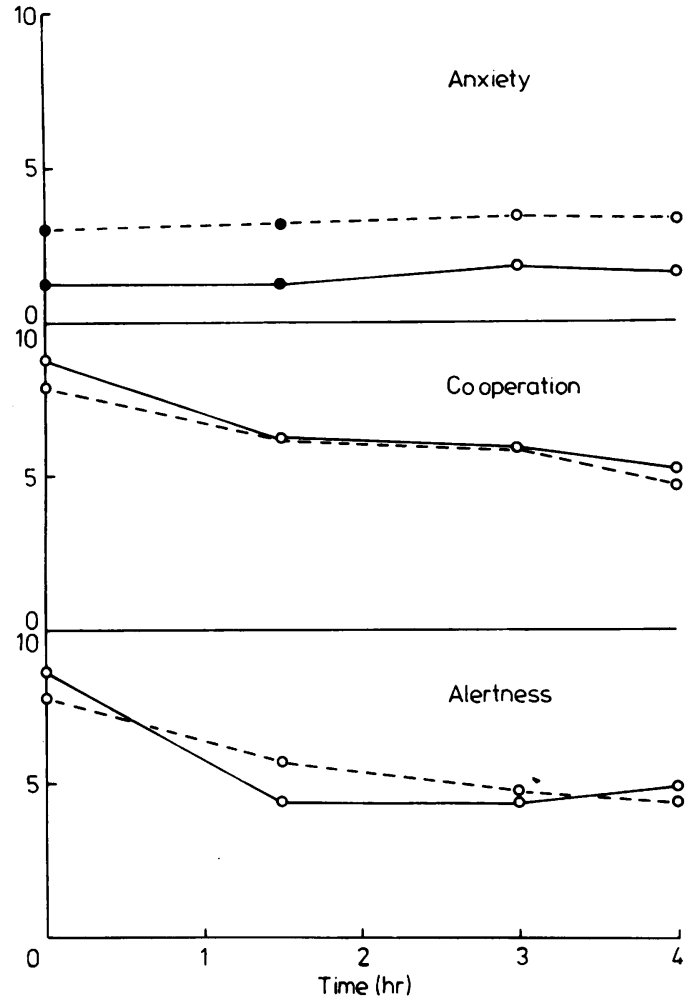

Fig. 2 Mean subjective ratings ( $\max 10)$ of anxiety, cooperation, and alertness for whole group during control $(0-0)$ and stress (O.... ) tape recordings. Closed circles $(\bullet)$ a significant difference $(p<0.05)$ between paired means.

mean breath hydrogen profile was similar for both studies, it was 'shifted to the left' in every case during stress.

\section{Discussion}

The dichotomous listening test used in our study seemed an appropriate stress because it attempted to mimic the experience of a busy person trying to perform several tasks at once, and being continuously interrupted by irritating or frustrating tasks. Moreover, it was comparatively simple to design a suitable control study and the fact that the subjects were studied in recumbent position, wearing headphones, meant that they were little affected by the activity in the laboratory. A further advantage was that the stress could be continued for several hours commensurate with the length of time taken for a meal to travel along the small intestine. Finally, the techniques used to measure the stress response were non-invasive and much less stressful 


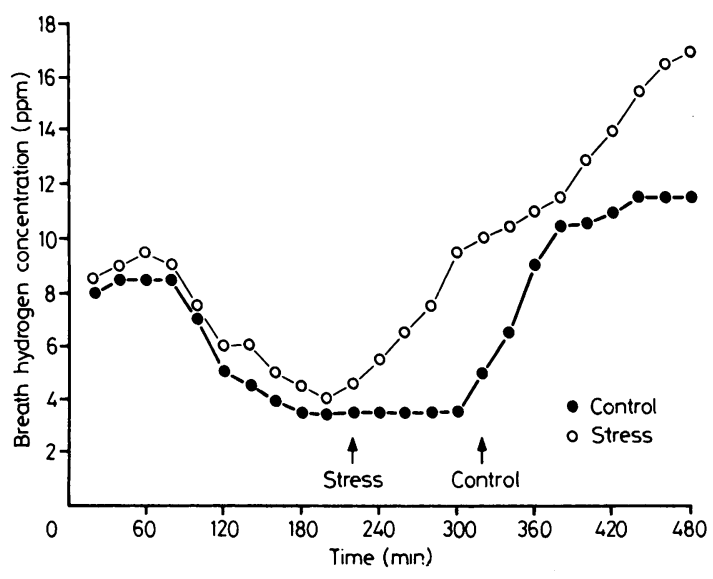

Fig. 3 Two graphs show the mean breath hydrogen levels for all subjects during control and stress studies respectively. Note similarity in profile of curves, but also that there was 'shift to the left' during stress studies with rise in breath hydrogen level (S) occurring well before rise during control studies $(C)$.

than the stress stimulus itself. The use of intestinal intubation to monitor a gastrointestinal response to stress can be extremely stressful in itself and could mask the effect of the experimental stress. We have previously found that intestinal intubation significantly delays gastric emptying and significantly accelerates small bowel transit time. ${ }^{28}$

All of our subjects cooperated with the protocol and each answered over $90 \%$ of the questions correctly during the dichotomous listening test. The test was associated with increases in both physiological and psychological indices of stress, although the levels of anxiety experienced by the subjects were generally low. This is perhaps not surprising as it is difficult to provoke and maintain high levels of anxiety for four hours with an experimental stress that approximates to a life situation and can be easily controlled.

Previous reports have suggested that the gastrointestinal response to stress varies according to the individual's emotional response. Fear and depression are associated with hypomotility and hyposecretion of the stomach ${ }^{11-13}$ and hypomotility in the small bowel ${ }^{17} 18$ and colon ${ }^{19} 20$ while resentment, anger, and aggression are associated with enhanced motor activity and secretion.

Our study shows that a prolonged mental stress that increased subjective and objective indices of anxiety induced a highly significant acceleration in small bowel transit, although it did not exert any significant effect on gastric emptying. These findings suggest that the rapid transit observed in patients with diarrhoea predominant irritable bowel syndrome $^{1}$ may be related to anxiety, particularly as only this group exhibits significantly higher anxiety ratings. ${ }^{2}$ This suggestion is supported by the previous observation that patients with 'functional bowel disorders', associated with stress and anxiety, have an increased propulsive activity in the small bowel. ${ }^{29}$ As such, our results are compatible with the hypothesis that abnormalities in IBS do not necessarily represent a primary constitutional impairment of function, but may be secondary to high levels of life stress or an anxious personality.

\section{References}

1 Cann PA, Read NW, Brown C, Hobson N, Holdsworth CD. The irritable bowel syndrome: relationship of disorders in the transit time of a single solid meal to symptom patterns. Gut 1983. (In press.)

2 Esler MD, Goulstan KT. Levels of anxiety in colonic disorders. N Engl J Med 1973; 288: 16-20.

3 Hislop IG. Psychological significance of the irritable colon syndrome. Gut 1971; 12: 452-7.

4 Creed F. Life events and appendicectomy. Lancet 1981; 1: 1381-5.

5 Chaudhury NA, Truelove SC. The irritable colon syndrome. $Q J$ Med 1962; 31: 307-22.

6 Murphy E, Brown GW. Life events, psychiatric disturbance and physical illness. Br J Psychiatry 1980; 136: $326-38$.

7 Almy TP, Fielding JF. The GI tract in stress and psychosocial disorder. In: Clinics in gastroenterology. London, Philadelphia, and Toronto: W B Saunders, 1977; 6: 3.

8 Bogdanoff MD, Bogdanoff MM, Wolf SG. Studies on salivary function in man: variations in secretory rate as part of the individual's adaptive pattern. $J$ Psychosom Res 1961; 5: 170-4.

9 Wolf SG, Almy TP. Experimental observations on cardiospasm in man. Gastroenterology 1949; 13: 40121.

10 Beaumont $\mathrm{W}$. Experiments and observations on the gastric juice and the physiology of digestion. (1833 Facsimile). New York: Dover Publications Inc, 1959.

11 Wolf S, Wolff HG. Human gastric function: an experimental study of a man and his stomach. 2nd ed. New York: Oxford University Press, 1947.

12 Bennett TI, Venables JF. The effects of the emotions on gastric secretion and motility in the human being. $\mathrm{Br}$ Med J 1920; 2: 662-3.

13 Engel GL, Reichman F, Segal HL. A study of an infant with a gastric fistula. Psychosom Med 1956; 18: 374-98.

14 Nagamachi Y, Taniguchi A. Effects of emotional stress on gastric secretion: its significance in the etiology of peptic ulcer. Jpn J Gastroenterol 1975; 72: 673-83. 
15 Granata L. Leone D. Paccione F. Changes in jejunal motility during emotional reactions. Arch Sci Biol 1973; 57: 87-97.

16 Wingate DL. McRae S. Younger K. Thompson DG. Stress and jejunal motor activity. (jut 1982: 23: $4(1+49$.

17 Cannon WB. The movements of the intestincs studied by means of Roentgen rays. Am J Physiol 19(1)2: 6: 251-77.

18 Friedman J. Roentgen studies of the effects on the small intestine from emotional disturbances. $A m ~ J$ Roentgenol Radium Ther Nucl Med 1954: 72: 367-79.

19 Almy TP. Kern F. Tulin M. Alterations in colonic function in man under stress. Gastroenterology 1949; 12: $425-36$.

20 Chaudhury NA. Truelove SC. Human colonic motility: effect of emotions. Gastroenterology 1961; 40: 27-36.

21 Read NW, Miles CA. Fisher D et al. Transit of a meal through the stomach, small intestine and colon in normal subjects and its role in the pathogenesis of diarrhea. Gastroenterology 1980; 79: 1276-82.

22 Stevenson NR, Sitren HS, Furuya S. Circadian rhythmicity in several small intestinal functions is independent of use of the intestine. Am J Physiol 1980; 238: G203-7.

23 Aitken RBC. Measurement of feelings using visual analogue scales. Proc Roy Soc Med 1969; 62: 989-93.

24 Lindsay PH, Norman DA. The phenomena of attention. In: Human information processing. 2nd ed. New York: Academic Press, 1977.

25 Metz G. Gassull MA, Leeds AR et al. A simple method of measuring breath hydrogen in carbohydrate malabsorption by end-expiratory sampling. Clin Sci Mol Med 1976; 50: 237-40.

26 Bond JH, Levitt MD. Investigation of small bowel transit time in man using pulmonary hydrogen measurements. J Lab Clin Med 1974; 84: 546-55.

27 Bergman I, Coleman JE, Evans D. A simple gas chromatograph with an electrochemical detector for the measurement of hydrogen and carbon monoxide in the parts per million range, applied to exhaled air. Chromatographia 1975; 8: 581-3.

28 Read NW, Al-Janabi MN. Small bowel intubation delays gastric emptying but accelerates small bowel transit time. Presented to the British Society of Gastroenterology, Norwich, March 1982. Gut 1982; 23: A443.

29 Horowitz L, Farrar JT. Intraluminal small intestinal pressures in normal patients and in patients with functional gastrointestinal disorders. Gastroenterology 1962; 42: 455-64. 